\title{
Efficient Calculation of a Jitter/Stability Metric
}

\author{
Daniel P. Giesy * \\ Guidance and Control Branch, NASA Langley Research Center, \\ $M / S$ 161, Hampton, VA 23681-2199
}

\begin{abstract}
A tool for computing a jitter/stability metric used in NASA requirements statements is developed. An efficient algorithm is given for computing this metric. Two ways of implementing it on a computer are discussed. One is optimized for computational speed while the other sacrifices some speed to conserve memory. Timing studies are given to show that the improvement of computation times using the present algorithm over previously existing techniques can run to several orders of magnitude, and that previous techniques were so costly that the present algorithm represents enabling technology. Further comparisons show that the memory conservative implementation runs at about half the speed of the fast implementation, but can cut the major data storage requirement of the fast implementation by $95-99 \%$, making the algorithm implementable on much smaller computers, such as PC's, than it would be otherwise. Software for both implementations is included in version 2 of the NASA time and frequency domain analysis program PLATSIM.
\end{abstract}

*Aerospace Technologist, Mathematician. The work in this paper was done while the author was a Computer Systems Specialist with Lockheed Martin and was published in the AIAA Journal of Spacecraft and Rockets, [1]. This work was supported by NASA Langley Research Center under contracts NAS1-19000 and NAS1-96014. 


\section{List of Figures}

1 A jitter window in four positions .......... 5

2 Potentially significant maxima and dominant element pointers before update . . . . . . . . . . . . . 11

3 Potentially significant maxima and dominant element pointers after update . . . . . . . . . . . . . . 12

4 Linear regression fit to window size timing data $\ldots \ldots \ldots 27$

\section{Nomenclature}

$a, b, c, d=$ Constants used in the jitter calculation timing formula, sec

$\mathrm{J}(\mathrm{y}, \mathrm{w}) \quad=$ See Definition 1

$k, k_{1}, \ldots, k_{m}=$ Number of data points in a time series sampled at equal time increments which are covered by windows $w, w_{1}, \ldots, w_{m}$

$l \quad=$ The number of different time signals being simultaneously analyzed by the memory conservative implementation of the algorithm

$l_{1}, \ldots, l_{m}=$ Dominant minimum pointers, same units as $t_{1}$, etc.

$m \quad=$ Number of windows for which jitter/stability is to be determined

$m_{i} \quad=$ Number of windows for which jitter/stability is to be determined in timing test case number $i$

$n, n_{i} \quad=$ Number of data points in a time series

$N \quad=$ An integer parameter used by the memory conservative implementation of the algorithm in managing dynamic memory allocation

$t_{1}, t_{2}, \ldots, t_{n}=$ Sample times for a discrete time series; arbitrary time units can be used, for a spacecraft instrument boresight pointing error application, typical unit would be sec

$T=$ Total duration of discrete time signal, i.e., $t_{n}-t_{1}$, in the same units as $t_{1}$, etc.

$u_{1}, \ldots, u_{m}=$ Dominant maximum pointers, same units as $t_{1}$, etc.

$w, w_{1}, \ldots, w_{m}=$ Lengths of jitter/stablility defining windows, same units as $t_{1}$, etc.

$y=$ Discrete time series of $n$ points; units are problem dependent, for a spacecraft instrument boresight pointing error measurement, typical unit would be arcseconds

$y(i) \quad=$ Same as $y\left(t_{i}\right)$, this notation only used if time points are equally spaced

$y\left(t_{i}\right) \quad=$ Element $i$ of time series $y$, same units as $y$

$y_{J}^{(w)} \quad=$ See Definition 1 


\begin{tabular}{|c|c|}
\hline$y_{L}^{(w)}$ & $=$ See Definition 1 \\
\hline$y_{U}^{(w)}$ & $=$ See Definition 1 \\
\hline$z_{i}$ & $=$ Calculation time per data point of test case $i$, sec \\
\hline Greek & \\
\hline & $=$ Execution time of test case number $i$, sec \\
\hline Subscripts & \\
\hline$i$ & $=$ Time point number, $1 \leq i \leq n$ \\
\hline$i$ & $=$ Test case number, $1 \leq i \leq 3808$ \\
\hline$j$ & $=$ Window number, $1 \leq j \leq m$ \\
\hline
\end{tabular}

\section{Introduction}

The purpose of this paper is to provide a computational tool whose uses include design and analysis of spacecraft. It presents an efficient method for calculating a metric that has been used by NASA to quantify requirements for pointing jitter and stability in spacecraft instrumentation [2]. This metric has been used to state requirements for the Upper Atmosphere Research Satellite (UARS), the Earth Observing Satellite (EOS), and individual instruments on these satellites. This jitter/stability metric has the advantage of having a high intuitive content; it is easy for an engineer to picture the connection between the jitter/stability value this metric assigns to any given time signal and the level of noise or drift in the signal. Since NASA states requirements in terms of this metric, it is important to be able to calculate it. However, anecdotal information ([3]) indicated that when attempts were made to calculate the jitter or stability of lengthy time signals, the computational burden was unacceptable. The computation was found to require anything from overnight or week-end computer runs in order to complete a single analysis to computations, such as the estimated 127 day example presented later, which were too computationally intensive to be feasible.

Another feature of this jitter metric is that its very definition immediately leads one to an easy algorithm for its computation. Anecdotal information [3] indicates that this was the algorithm in use when unacceptable computational burdens were encountered. The problem is that this algorithm is not the most efficient way to calculate this jitter metric. The exact degree of inefficiency depends on the signal length, the sampling frequency, and another problem parameter (the window length); but problems of engineering interest have been found for which the easy algorithm is three 
or four orders of magnitude slower than the algorithm to be presented in this paper. The penalty paid for the additional computational speed of the new algorithm is a vast increase in algorithmic complexity.

Examples were this metric has been used can be found in industry working papers $([4,5])$, in conference proceedings $([6,7])$; and an occasional mention can even be found in the journal literature ([8]). Reference [6] says, "Jitter ... requirements [are] expressed as a worst-case change in pointing ... across each time interval of interest ...." In reference [5] "Pointing Jitter" is defined to be "the peak-to-peak variation of the actual pointing direction over relatively short time intervals" with "Pointing Stability" using the same definition except that the time intervals are "relatively long."

In this paper, the "time intervals" used as a parameter in the definition of the jitter/stability metric will be called windows. Then the jitter or stability value of a given time signal with respect to one of these windows is found by placing the window over the time signal in such a position as to maximize the peak-to-peak variation of that portion of the signal which is under the window. It is this maximum peak-to-peak variation which is taken as the value of the metric. Since the same algorithm is used to calculate either the jitter metric or the stability metric, the word "jitter" will be used for the remainder of this paper to refer to either jitter or stability as defined above.

The technique presented in this paper can be applied to an arbitrary discrete function of finitely many time points. The units will be determined by the specific use which is being made of it. For quantifying the jitter in the boresight pointing error of an observational instrument on a spacecraft, typical units for time might be seconds while units for the boresight error might be arcseconds. On the other hand, if one is looking at irregularities in tectonic plate drift, the units might well be centuries and centimeters. So, for the most part in this paper, the units will be left unstated.

Fig. 1 shows one such window in four of its possible positions on a discretely sampled function of time. The length (duration) of each time window, represented by the width of the rectangle, is the same for all four window positions. The top and bottom of each rectangle is positioned to show the peak-to-peak variations of the function within the window. The tallest rectangle represents one window position where the peak-to-peak variation reaches its worst case value, so its height represents the jitter in this time function for this window size. Note that the leftmost window extends into the negative part of the time axis where there are no data points from the discrete 


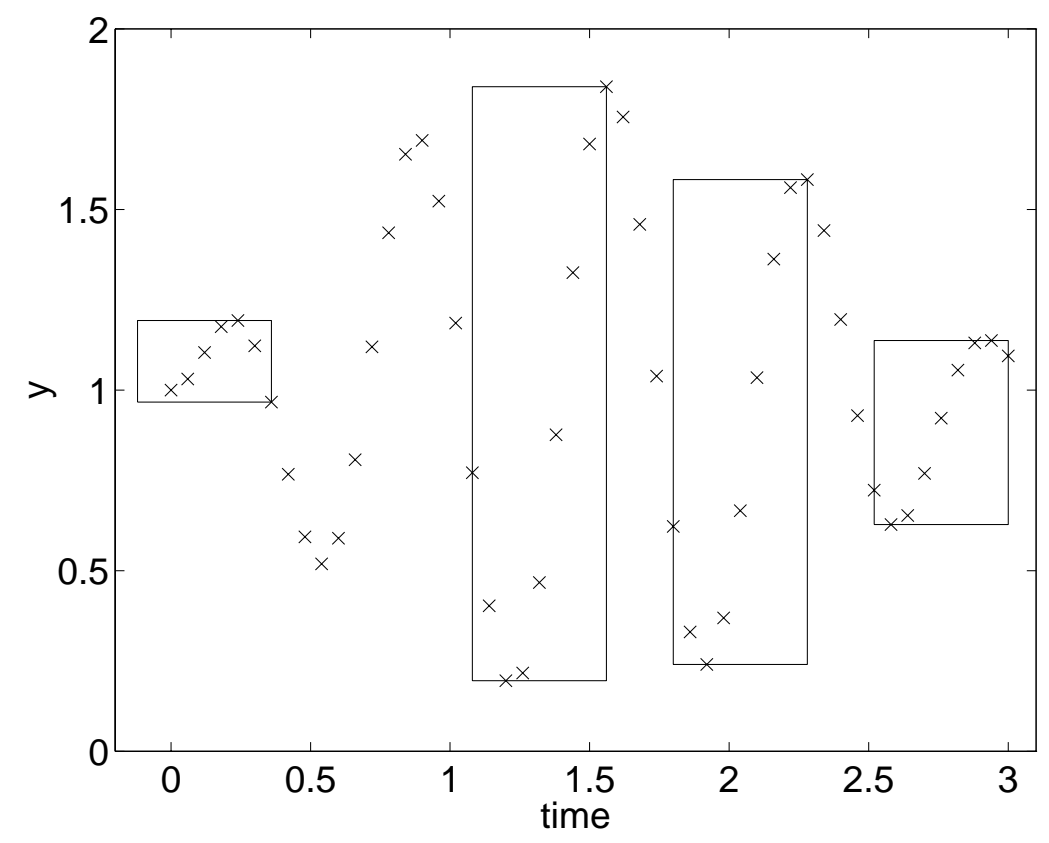

Figure 1: A jitter window in four positions

time signal. Including such windows does not change the value calculated for the jitter metric, and will be useful in implementing the algorithm.

In order to calculate this jitter metric with respect to a given window, a position must be found for this window which maximizes the peak-to-peak variation of the time signal under the window. The easy algorithm to accomplish this calculation is to simply try all window positions and pick the worst case from among them. If the signal is discrete and of finite length (discrete signals of finite length can arise if the data come from sampling the output of some sensor or if the data are generated by a numerical simulation), then there are only a finite number of window positions with the property that any two of them cover different sets of points; so this is an algorithm which can be implemented on a computer. This search can be performed in a systematic fashion by first placing the window at the beginning of the time signal and calculating the peak-to-peak variation under it, then moving the window forward in time until it covers one additional point of the time signal and repeating the peak-to-peak variation determination, and continuing to advance the window one point at a time and determine the new peak-to-peak variation until the end of the signal is reached. The measure of jitter is then 
the worst of these peak-to-peak variations. This is easily programmed in FORTRAN using a nested pair of DO loops or in MATLAB (MATLAB is a registered trademark of The MathWorks, Inc.) using a vectorized determination of the peak-to-peak variation under the window in a given position inside a single for loop.

If the data points in a discrete signal are equally spaced, the computational complexity of the jitter calculation for each window is determined by two parameters: $n$, the number of points in the data sequence; and $k$, the number of points of data that lie under the chosen window. These numbers can be quite large. For example, the data may come from a simulation driven by disturbances with high-frequency components, so that accuracy requires an integration interval which is small compared to the window size, resulting in a large value for $k$. A small integration interval will also drive up the value of $n$, as will a need to consider long term effects.

If the calculation of jitter is programmed on a computer using the easy algorithm, the time to calculate the peak-to-peak variation of the signal under the window in one position is $O(k)$, and $n-k+1$ window positions must be considered, so the total calculation time is $O(k(n-k+1))$. If $n$ is large and $1 \ll k \ll n$ (in one jitter analysis of the EOS AM-1 spacecraft [7], values of $n$ and $k$ on the order of $10^{5}$ and $10^{4}$, respectively, were common), this takes a substantial amount of time. In an analytic jitter study, the computational expense can be driven up by such factors as: (a) Each output signal may need to be analyzed over several different windows (4-7 in the EOS AM-1 study); (b) For each disturbance scenario, many output signals need undergo the Step (a) analysis (20-30 in the EOS AM-1 study; representative times for a jitter calculation at this level using both the easy algorithm and the one given in this paper are given in Table 1); (c) Step (b) must be repeated for many disturbance scenarios (over 20 in the EOS AM-1 study); (d) Step (c) must be repeated for each design iteration. At this point, the need for a more efficient jitter calculation should be apparent. At the same time, approximation techniques should be avoided if possible.

The purpose of this paper is to present a much more efficient algorithm which exactly calculates the NASA jitter metric. This algorithm can be used to simultaneously calculate jitter in a time series for each of several windows. A list is maintained of potentially significant maxima and another of minima as the time series is scanned. At each time step, information from these lists is used to update variables which accumulates the metric of jitter for each window, leading, at the end of the scan, to a determination of jitter values 
for all windows. If there are $m$ windows, the time to do this is $(a m+b) n$ where the constants $a$ and $b$ are independent of the actual window sizes. The calculation time for the first window is $(a+b) n$. Comparing this to the timing estimate $O(k(n-k+1))$ for the easy algorithm, it is seen that the removal of the dependency on the window length $k$ has replaced a timing formula which is jointly quadratic in $n$ and $k$ by one which is linear in $n$ and independent of $k$. The incremental calculation time for each additional window is an. This incremental time for each additional window has been empirically observed to be about $30 \%$ of the time for the first window, so the computational time to calculate jitter for several windows simultaneously is substantially better than doing the calculation for each window individually.

This algorithm has been implemented in software and included in version 2 of the PLATSIM software package $([9])$. PLATSIM is a MATLAB based software package, developed at NASA Langley Research Center, which performs time and frequency domain analysis of the response of a controlled or uncontrolled flexible structure to disturbances. Version 1.0 of PLATSIM ([10], [11]) contains an earlier version of this algorithm (without the memory conservative option).

First, the algorithm is given. Then, notes on two implementations of the algorithm are presented. The first implementation emphasizes computational speed while the second implementation sacrifices some computational speed in order to conserve computer memory. Results of timing studies to quantify the improvements in computational time and to show the dependency of timing on problem parameters are shown. A few remarks on the amount of memory saved using the memory conservative implementation of the algorithm are made. The paper ends with a section of conclusions.

\section{The jitter calculation algorithm}

In this section the problem is dealt with of calculating the jitter in a time series over one or more jitter windows. Although the windows under which the maximum peak-to-peak excursions of the time series are being observed are thought of as sliding continuously down the time axis; for purposes of determining jitter, a window need only be considered when it is positioned so that its right end is coincident with one of the points of the time series. (A window in this position is referred to as being in standard position; the windows shown in Fig. 1 are placed in standard position.) This can be seen by observing that if a window in standard position starts sliding 
to the right, the maximum peak-to-peak excursion under that window cannot increase until a new point of the time series comes into the window. This happens exactly when the window reaches the next standard position. So, no jitter information is lost by ignoring the window in any of the intermediate positions.

Notation Let $n$ be the number of points in the time series and denote the time series by $\left\{y\left(t_{i}\right) \mid 1 \leq i \leq n\right\}$. Assume that $t_{1}<t_{2}<\cdots<t_{n}$. Suppose that there are $m$ windows and they have lengths $w_{1}<\cdots<w_{m}$. If the time points are equally spaced, the time series might be written as $\{y(1), y(2), \ldots, y(n)\}$ and $k_{j}$ used to denote the number of points covered by a window of length $w_{j}$ in standard position.

In Fig. 1 , a time series is shown with $n=51, t_{1}=0$, and $t_{n}=3$. One window is shown (in four different positions), so $m=1$. For this window, $w_{1}=.48$, and so $k_{1}=9$.

Definition 1 For a window of length $w$, denote the running tallies of the upper and lower bounds of $y$ under each window position by $y_{U}^{(w)}$ and $y_{L}^{(w)}$ and define them by

$$
\begin{aligned}
& y_{U}^{(w)}\left(t_{i}\right)=\max \left\{y\left(t_{j}\right) \mid t_{i}-w \leq t_{j} \leq t_{i}\right\} \\
& y_{L}^{(w)}\left(t_{i}\right)=\min \left\{y\left(t_{j}\right) \mid t_{i}-w \leq t_{j} \leq t_{i}\right\}
\end{aligned}
$$

where

$$
1 \leq i \leq n
$$

Denote the jitter time history of $y$ for this window by $y_{J}^{(w)}$ and define it by

$$
y_{J}^{(w)}\left(t_{i}\right)=y_{U}^{(w)}\left(t_{i}\right)-y_{L}^{(w)}\left(t_{i}\right), \quad 1 \leq i \leq n
$$

Finally, denote the jitter by $J(y, w)$, and define it by

$$
J(y, w)=\max \left\{y_{J}^{(w)}\left(t_{i}\right) \mid 1 \leq i \leq n\right\}
$$

Where the window length is clear from context, the superscript $(w)$ will be omitted. 
In Fig. 1, the windows shown correspond to $i=7,27,39$, and 51. The numbers $y_{U}\left(t_{i}\right), y_{L}\left(t_{i}\right)$, and $y_{J}\left(t_{i}\right)$ represent, respectively, the top $\mathrm{y}-$ coordinate, the bottom y-coordinate, and the height of the corresponding rectangle. Note that even though $k=9$, the leftmost pictured window covers only 7 points; its left side extends into negative time where the time series is not defined.

One easily sees that, in Eq. (5), the same value will be computed for $J(y, w)$ if the lower limit of 1 is changed to a larger number, so long as $t_{1}$ lies under the first window used. If jitter were to be calculated using the easy algorithm, this lower limit would be $k$, the number of points covered by a window of length $w$ positioned at the beginning of the time series. Doing the calculation by using the easy algorithm refers to actually doing the calculations in equations Eqs. (1), (2), (4), and (5). Referring to Fig. 1, if one wished to calculate jitter using the easy algorithm, it would be inefficient to use the first window position shown there. It would be more efficient to start with a window positioned with its left edge at time 0 . If the time steps are evenly spaced so that there are $k$ points under a window in standard position, then doing the calculations in equations Eqs. (1) and (2) requires $k$ references into the $y$ vector for each entry in $y_{U}$ and $y_{L}$. Since the first $k-1$ entries of $y_{J}$ are dominated by $y_{J}(k)$, these need not be computed, and only the last $n-k+1$ values of $y_{U}$ and $y_{L}$ are needed.

The comparative speed of the algorithm to be presented in this paper can now be explained. When jitter is calculated using the easy algorithm, then for typical values of $n$ and $k$, most entries in $y$ are being referenced $k$ times. The algorithm presented here reduces the number of references per time series entry from $k$ references (for most time series elements) to a small number of references which is bounded independent of $k$. Some overhead is incurred on each pass through a time series. This is amortized over all the windows being considered in the pass, so the bound on the number of times a given series element is referenced per window improves as more windows are considered simultaneously.

If one only wishes to calculate $J(y, w)$, it is not necessary to spend computer memory to contain the full arrays $y_{U}, y_{L}$, and $y_{J}$. For each $i$, one could calculate the $i$-th element of each of these arrays, accumulate the necessary information from this calculation in the jitter-to-date tally, and discard these $i$-th elements.

The following discussion details the calculation of $y_{U}\left(t_{i}\right)$. The calculation of $y_{L}$ is parallel. One need only reverse the direction of inequalities in- 
volving elements of $y$ and replace "decreasing" by "increasing" in statements about the monotonicity of potentially significant extrema lists of elements of $y$.

The outer loop of the easy algorithm can be thought of as making a pass through the time signal. Each step of this pass places the scanning window in a new position. The inner loop then scans every element of the time signal under the window to determine the maximum and minimum so the peak-to-peak variation can be computed. If this calculation needs to be done for more than one window, the calculation for each window is traditionally done independently of that for the others.

The present algorithm also makes a pass through the $y$ vector. However, instead of scanning the length of a window back from the current position to determine the maximum and minimum under that window, the information needed to find that maximum and minimum has already been recorded in some bookkeeping arrays in a manner which allows for efficient retrieval and update. Another advantage to this technique is that the jitter calculation can be done for several windows simultaneously at less computational cost per window than performing the calculation for each window individually. Two lists are being maintained, one a list of potentially significant maxima and the other a list of potentially significant minima. The potentially significant maxima (respectively, minima) list contains elements of the $y$ vector which have already been scanned and which have the potential of being the maximum (respectively, minimum) element in one of the windows in the present and/or some future position of the pass. Each window has three bookkeeping items associated with it, a jitter-to-date tally, a dominant maximum pointer, and a dominant minimum pointer. The jitter-to-date tally records how much jitter there is in the time series up to the point most recently scanned. The dominant maximum pointer points at the location in the list of potentially significant maxima which gives the maximum value of the time series under the window when its right end is at the current element of the pass. There is an analogous relationship between the dominant minimum pointer and the list of potentially significant minima. All of these bookkeeping elements must be updated with each step through the $y$ vector. The efficiency of the algorithm comes in that this update can be accomplished in an average time bounded by an expression of the form $a m+b$ where $a$ and $b$ are absolute constants which are, in particular, independent of the lengths of the individual windows.

The process of making one step through the $y$ vector can be thought of 
as occurring in the following sequence: Initially, all windows have their right endpoints at the last element of the $y$ vector which has been scanned; for each window, the jitter in $y$ up to the last scanned point has been entered into a jitter-to-date tally; all the information in the potentially significant extrema lists is relevant to windows in this position and future positions; and the dominant element pointers are correct for this window position. Then, the next point of the $y$ vector is scanned and the windows all slide from their old position to their new position with their right endpoints all coincident with the time at which the new $y$ point occurs. Then, the new significant local extrema lists are formed from the old. Then, the dominant element pointers are updated to be correct for the new window position. Finally, the jitter-to-date tally for each window is updated with new information from this step.

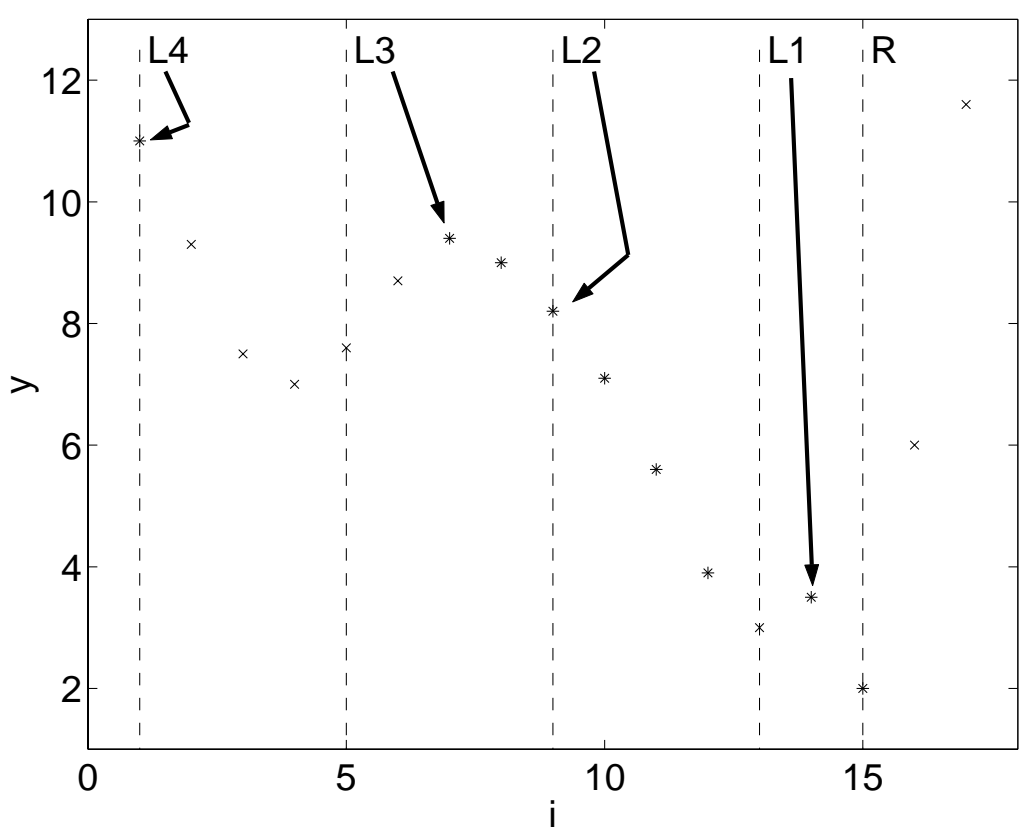

Figure 2: Potentially significant maxima and dominant element pointers before update

The process of advancing the calculation of $y_{U}$ by one time step can be observed from Figs. 2 and 3. In both figures, the same generic time sequence $y$ is shown. It is sampled at 17 equally spaced points, so $n=17$. There are 


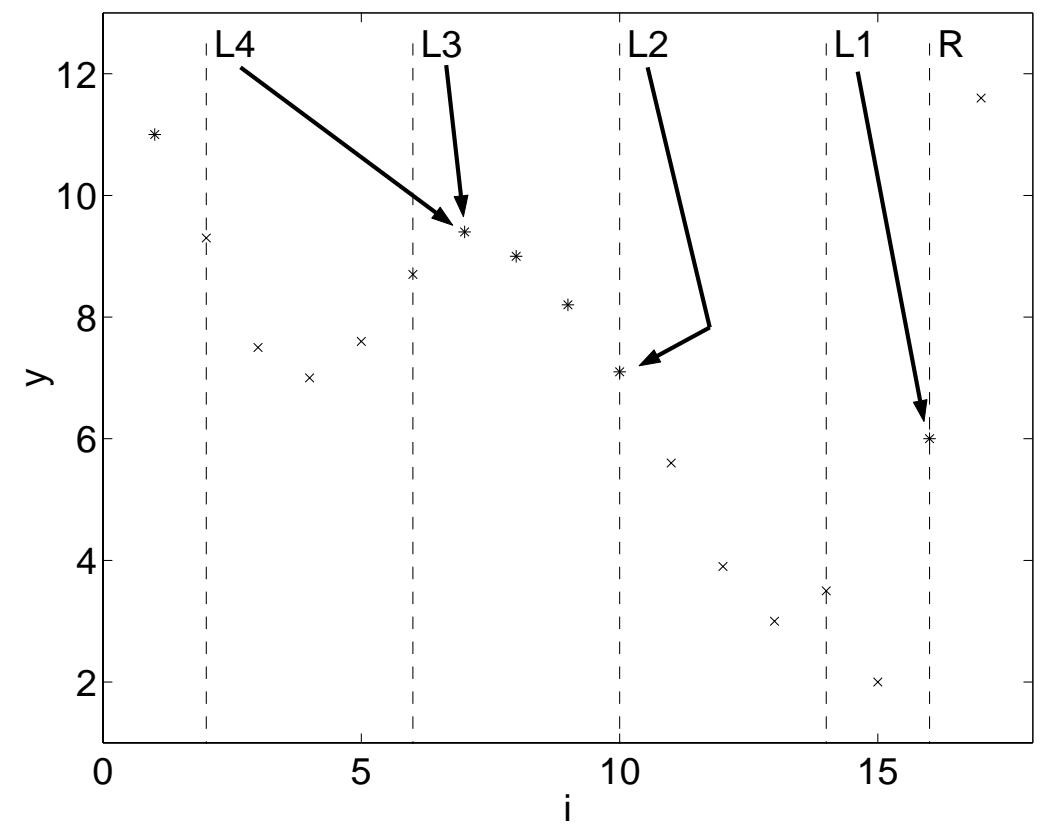

Figure 3: Potentially significant maxima and dominant element pointers after update 
four windows with respect to which jitter is being calculated $(m=4)$. The window lengths are given by the point counts $k_{1}=3, k_{2}=7, k_{3}=11$, and $k_{4}=15$. Fig. 2 shows the situation in computing $y_{U}$ after the scan of $y$ has reached $y(15)$ while Fig. 3 shows the situation after reaching $y(16)$. The four windows overlap, having a common right edge at the dashed line labeled $\mathrm{R}$. The left edge of $w_{1}$ is at the dashed line labeled L1, the left edge of $w_{2}$ is at L2, and so on. Potentially significant maxima are shown as stars, other data points as x's. Dominant element pointers are shown for each window. Thus, $y_{U}^{\left(w_{1}\right)}(15)=y(14), y_{U}^{\left(w_{2}\right)}(15)=y(9)$, etc.

At the point of the calculation shown in Fig. 2, the algorithm has only seen $y(1), \ldots, y(15)$ and it is computationally efficient for the algorithm to assume that the series goes on indefinitely. One possibility the algorithm must consider is that the series is monotone decreasing for at least another 14 points. If that is the case, every one of the potentially significant maxima is either the maximum element in one of these windows in the present position (these are marked by the dominant element pointers) or will become the maximum element under one or more windows at some future position (or both). The possibility that it might become a maximum in some future window is the potential significance of each of these elements in computing the maxima of the series $y$ over some jitter windows. Note that the subsequence of $y$ formed by the potentially significant maxima is monotonically decreasing and that each dominant element pointer points to the left-most potentially significant element under its window. This always happens, and can be established by induction on $i$.

Now observe what happens when the scan of $y$ advances to $i=16$ in Fig. 3. The newly scanned element always becomes potentially significant. In this example, it is bigger than several of the potentially significant maxima at the previous stage (located at $i=15,14,12$, and 11), so they lose their potential significance. Note that the demoted elements were placed consecutively at the right end of the old list of potentially significant maxima. This always happens; and because of this, the demotion of previously potentially significant extrema is an efficient computation. The dominant element pointers are updated. For window 1, the previous dominant element lost significance, so the pointer was redirected to the newly scanned element $y(16)$. For windows 2 and 4, the previous dominant element dropped out of the left end of the window as it moved on, so the pointer was moved to the right in the list of potentially significant maxima until an element was found in the window. Since points are equally spaced, the pointer needed only to 
move to the next element in the list of potentially significant maxima. For unequally spaced points, several steps might have been necessary. The third window could leave its dominant element pointer where it was the previous step. Note that at this point, the same element, $y(7)$, is the maximum under both the third and fourth windows.

The reader should note that in one more step $(i=17)$, the only potentially significant maximum will be the new point $y(17)$, and all dominant element pointers will point to it.

The efficiency of the algorithm comes from the way in which the lists of potentially significant extrema and the dominant element pointers are updated from step to step. As another step is taken, the new element from the $y$ vector is potentially significant as both a maximum and minimum. Whether either of these potentials is realized will depend on future behavior of the $y$ vector. So the new element will be added to the end of both lists. What is important is that the presence of this new element on a list may remove any potential significance which some of the previous elements had, so they are deleted from the list.

Specifically, when a new element is added to the list of potentially significant maxima, then any element already on the list which is less than or equal to the added element is no longer potentially significant. This loss of potential significance is seen by observing that a window in its new position or in any future position which covers the earlier, dominated, element also covers the element just added, so the earlier element is not needed to calculate the peak-to-peak excursion in any such window. Thus, the list of potentially significant maxima will be updated by removing all elements less than or equal to the new element and then adding the new element at the end of the resulting list. Consequences of updating the list in this way are that the elements in this list form a monotonically decreasing sequence, and that the set of elements deleted from the list as the result of the new addition are located consecutively at the end of the pre-update list. These consequences may be demonstrated by induction on the position of the scan in the $y$ vector (at the initial step, the list contains a single element and so is vacuously monotonic decreasing). The list of potentially significant minima is updated similarly.

Since the list of potentially significant maxima is a decreasing sequence, the dominant maximum pointer for each window points as far to the left in the list as it can be and still have the element it points to lie in the window in its present position. This characteristic of the position of the 
dominant maxima simplifies the process of updating their pointers after each step.

The dominant element pointers are updated next. For each pointer, there are three possibilities. The first possibility is that the element being pointed to by a given window's pointer may have been removed because it is dominated by the new element. Then the pointer is redirected to this new element. The second possibility is that the element being pointed to by a given window's pointer may no longer be in that window after the window has moved to its new position. This possibility happens when, before the update, the dominant element was at the left end of the window. In this case, the potentially significant element list is scanned starting at the old pointer position and moving to the right until an element is found which is in the window in its new position. If the time points are equally spaced, it is only necessary to move one element down the list. Third, if neither of the two previous circumstances hold, the pointer is left where it was.

For a given window, the maximum and minimum of $y$ over the window in its new position are determined by the elements in the potentially significant extrema lists pointed to by the window's dominant element pointers. From these values, the peak-to-peak excursion of $y$ over the window in this position can be determined. This is compared to the jitter-to-date tally for this window, and if the new peak-to-peak excursion is greater, the tally is updated with this value.

Once the step involving the last point of $y$ has been completed, the desired jitter values are found in the jitter-to-date tallies. This informal algorithmic description is formalized in algorithmic language as follows:

Algorithm 1 Step 0. Initialization. Initialize the potentially significant maxima and minima lists to $\left\{y\left(t_{1}\right)\right\}$. For $j=1,2, \cdots, m$, set $y_{U}^{\left(w_{j}\right)}\left(t_{1}\right)=$ $y_{L}^{\left(w_{j}\right)}\left(t_{1}\right)=y\left(t_{1}\right)$, and set $y_{J}^{\left(w_{j}\right)}\left(t_{1}\right)=J\left(y, w_{j}\right)=0$. Set the iteration counter, $i$, to 1 and set all the dominant maximum pointers $u_{1}, \cdots, u_{m}$ and all the dominant minimum pointers $l_{1}, \cdots, l_{m}$ to $t_{1}$.

Step 1. Increment $i$ by 1. If $i>n$, terminate; the desired jitter values are in $J\left(y, w_{1}\right), \ldots, J\left(y, w_{m}\right)$. Otherwise, continue.

Step 2. Start scanning the potentially significant maxima list from right to left. Each time a scanned element is less than or equal to $y\left(t_{i}\right)$, remove it. Stop the scan the first time an element is encountered which 
is greater than $y\left(t_{i}\right)$ or when the list becomes empty. Perform the same operation on the potentially significant minima list reversing the inequalities.

Step 3. Add $y\left(t_{i}\right)$ to the right end of both potentially significant extrema lists.

Step 4. For $j=1,2, \cdots, m$;

Step 4.1 Update the pointers to the dominant maxima and calculate $y_{U}^{\left(w_{j}\right)}\left(t_{i}\right)$ :

(a) if $y\left(u_{j}\right)$ was removed from the potentially significant maxima list at Step 2, set $u_{j}=t_{i}$; otherwise,

(b) if $u_{j}<t_{i}-w_{j}$, then scan the potentially significant maxima list starting at $y\left(u_{j}\right)$ and moving to the right until the first element $y\left(t_{s}\right)$ is found for which $t_{s} \geq t_{i}-w_{j}$, and set $u_{j}=t_{s}$; otherwise,

(c) $u_{j}$ remains the same.

Then set $y_{U}^{\left(w_{j}\right)}\left(t_{i}\right)=y\left(u_{j}\right)$.

Step 4.2 Similarly, update the pointers to the dominant minima and calculate $y_{L}^{\left(w_{j}\right)}\left(t_{i}\right)$.

Step 5. For $j=1,2, \cdots, m$, set $y_{J}^{\left(w_{j}\right)}\left(t_{i}\right)=y_{U}^{\left(w_{j}\right)}\left(t_{i}\right)-y_{L}^{\left(w_{j}\right)}\left(t_{i}\right)$ and if $y_{J}^{\left(w_{j}\right)}\left(t_{i}\right)>$ $J\left(y, w_{j}\right)$, then set $J\left(y, w_{j}\right)=y_{J}^{\left(w_{j}\right)}\left(t_{i}\right)$.

Step 6. Return to step 1.

Note: If time points are equally spaced, then the bookkeeping in this algorithm can be reduced by using the subscript, $i$, instead of the time value, $t_{i}$, for the dominant element pointers and using the number of points, $k_{j}$, under a window instead of its length in seconds, $w_{j}$, in the Step 4.1(b) and 4.2 (b) tests.

Starting the algorithm at $i=1$ would be wasteful if jitter were being calculated using the easy algorithm. However, in this algorithm, it serves the very useful purpose of properly initializing the potentially significant extrema lists and dominant element pointers, and it simplifies the additional bookkeeping necessary for tracking multiple windows simultaneously. 
Remarks on cost: For the most part, these remarks address the case of equal time increments. The time required to calculate jitter using the easy algorithm for a single window covering $k$ points would appear to be proportional to number of times an element of the $y$ array is referenced. Thus, the time to calculate jitter using the easy algorithm is proportional to $k(n-k+1)$. If the time series $y$ has duration $T$ (e.g., if $y(1)$ occurs at time 0 and $y(n)$ occurs at time $T$ ) and the window has length (duration) $w$, then $k$ is the smallest integer such that $k \geq(n-1) w / T$. So, for fixed values of $T$ and $w$, the time to calculate jitter using the easy algorithm is a quadratic function of the number of points, $n$, used in discretizing the time interval. The coefficients of this quadratic function depend on the length, $w$, of the window. The total time needed to calculate jitter with respect to several windows is found by adding up the times to calculate jitter for each individual window.

On the other hand, an examination of Algorithm 1 shows that the time to calculate jitter using this algorithm may be bounded by an expression of the form $a m n+b n+c m+d$ where $a, b, c$, and $d$ are absolute constants. The $c m+d$ part of this comes from Step 0 of the algorithm and, in any realistic example, is an inconsequential part of the total time. The total time for Step 1 is proportional to $n$. The time in Step 2 is spent either in removing elements from lists of potentially significant extrema or in examining an element of one of the lists and discovering that it stops further removal. Since a given element of $y$ can be removed from a list only once during the entire course of the jitter calculation, the "remove" part takes time proportional to $n$. Since it only takes one element per step to stop a removal back scan, this part of Step 2 also takes time proportional to $n$ as does Step 3. Step 4 is executed $n-1$ times, so Step 4.1 executed is $m(n-1)$ times. Most of the parts of Step 4.1 require an execution time which can be bounded by a fixed amount. The only exception is the part of substep (b) referring to a scan of elements in a potentially significant extrema list, and that only when the $y$ points are unequally separated in time. But here, considering the entire jitter calculation, a given element of $y$ can only be scanned once per window per list. Thus, the total time spent on Step 4.1 is $O(m n)$. Exactly the same considerations apply to Step 4.2. Similarly, the time for Step 5 is $O(m n)$ and for Step 6 is $O(n)$.

Comparing the two times, it is seen that the the easy algorithm timing expression is a sum of $m$ quadratics in $n$, so can be thought of as being $O\left(m n^{2}\right)$, while the timing expression for the present algorithm is $O(m n)$. For 
large $n$, the savings potential is great. The extent to which that potential is realized will be detailed in a subsequent section.

\section{Implementation notes}

Two implementation scenarios are considered. In the first, it is assumed that the complete time series $y$ is available to the jitter algorithm at each step in the scan. Thus, the jitter analysis code can keep track of any past information it needs from $y$ by an indirect addressing scheme into the $y$ vector itself. This first scenario produces the faster jitter analysis code. In the second, it is assumed that the points of $y$ are fed to the jitter analysis code one at a time and that the jitter analysis code has complete responsibility for any necessary recall. The emphasis in this latter case will be on managing computer memory to keep memory usage within reasonable bounds.

While programming for speed of execution hardly needs justification, in this era of multi-megabyte computer memories the need for care in memory usage may. Consider, then, a time simulation involving a 1 kilohertz sample rate extending over a 1000 second duration. Suppose that 50 different outputs must be analyzed for jitter. These numbers are within the bounds of parameters that have arisen in actual applications of this technology. Then, each output time history contains 1,000,001 double precision numbers, which will be assumed to occupy the IEEE standard of 8 bytes of computer storage. Taking into account that all 50 output time histories must be generated and stored at one time (running multiple simulations to generate the output time histories in smaller groups has its own penalty in terms of execution time) it is then seen that just storing these $50 y$ 's requires over 381 megabytes of computer memory (recall that computer manufacturers use "megabyte" to refer to $2^{20}$ bytes, not $10^{6}$ ). While it might be possible to equip a high-end workstation to accommodate this in physical memory, a much more common result of trying to run a problem this size would be that a lot of virtual memory paging would be necessary. This would cause a severe increase in wall-clock time of execution.

\section{The Fast Implementation}

In this scheme, multiple outputs are analyzed one at a time so that the arrays used in potentially significant extrema processing can be reused for each output. It will be seen that this prevents what would otherwise be a doubling of storage needed (assuming that an integer requires half the storage of a double precision number) to hold just the output time histories. 
The potentially significant extrema lists are realized by arrays of integers which point to the appropriate elements of the $y$ vector (i.e., an indirect addressing scheme). These arrays must be the same length as the $y$ vector. If $y$ is strictly decreasing in time, then in Step 2 of Algorithm 1, no elements of $y$ are ever removed from the potentially significant maxima list, so it must be big enough to hold them all. An increasing $y$ imposes the same restriction on the list of potentially significant minima. For each list, there is an integer variable containing a pointer to the current active right end of the list, and an array of $m$ integers which contain pointers to the place in the list pointing to the dominant elements for each window (in effect, a doubly indirect addressing scheme into the $y$ vector). One of the efficiencies of the algorithm comes from the fact that once an entry is made into one of these potentially significant extrema pointer lists, it is never moved (although it may be overwritten). "Step 2" removal of dominated elements is accomplished by redefining the "right end" pointer to point farther to the left. "Step 3" is accomplished by incrementing the "right end" pointer by 1 and storing the current value of $i$ in the new right end of the list. The "Step 4.1(a)" decision can be made by comparing the dominant element pointer to the new "right end" pointer and the "Step 4.1(b)" decision can be made by comparing the value pointed to by the dominant element pointer, the current value of $i$, and the number of points in the relevant window. Similar remarks apply to Step 4.2. The information needed to complete "Step 4" and do "Step 5" comes from following the dominant element pointers into the lists of pointers to potentially significant extrema and following those pointers into the $y$ vector.

This scheme has been implemented in FORTRAN 77 code subroutines. Further code provides an interface to MATLAB. All this code is compiled into a MATLAB MEX-file, which is a file of compiled binaries which can be called directly from MATLAB. In this form, it is part of the PLATSIM, Version 2.0, software package [9].

\section{The Memory Conservative Implementation}

In order to keep memory usage to a relatively minimal level, dynamic memory management such as is available in the $\mathrm{C}$ programming language is employed. The scheme must be capable of calculating the jitter in several time series (e.g., multiple output channels) simultaneously.

For each time series, the potentially significant maxima list and the potentially significant minima list will be maintained using a doubly linked 
linear list of storage structures. By a "storage structure" is meant a data storage construct (such as the $\mathrm{C}$ programming language data type struct) which can hold several items or "fields" of data, possibly of dissimilar types. What makes a collection of these structures a "doubly linked linear list" is the logical organization of this set of structures into a linear ordering with one being thought of as the first (pictured as the leftmost) possibly followed by a second, a third, and so forth until a last (the rightmost) is reached. The programming mechanism for this logical ordering is the inclusion of two fields in each structure, one of which contains a pointer to the structure immediately to its left (or a special marker if the structure is at the left end of the list) and the other of which contains a pointer to the structure immediately to its right (or a special marker if the structure is at the right end of the list). Each storage structure must be able to store the dependent value $y\left(t_{i}\right)$ of an element of the time series and also the time $t_{i}$ or, in the case of equally spaced points, the time index $i$ at which the time series takes on this value.

A first-in, first-out list of these structures will be maintained as a reserve stack to provide new structures as needed and on which to place unneeded structures as the information in them becomes obsolete.

For each potentially significant extrema list, one pointer will keep track of its right end and, for each window, an additional pointer will keep track of the leftmost structure in each list which contains information from under this window (these correspond, respectively, to the "right end" pointer and the "dominant element" pointers of the Fast Implementation). As each new step in the jitter calculation is taken, each list will be updated using a new data point from one of the time series. Any structures containing information obsoleted by the new value will be moved to the input end of the reserve stack. Structures will be moved from the output end of the reserve stack to the right end of each potentially significant extrema list to hold the new value. The "right end" pointers will be updated to point to the structures just added, and the "dominant element" pointers will be updated as necessary.

It is important to realize that when a structure is spoken of here as having been "moved" from one list to another, this does not mean that there has actually been a movement of data from one part of physical computer memory to another. What is altered are the values of various pointers which define the position of the structure in one or another of the logical lists.

If there are $l$ time signals being jitter analyzed, then $2 l$ structures 
must be moved from the reserve stack at each jitter calculation step. Before that step is taken, a check will be made of the number of structures in the reserve stack. If it is fewer than $2 l+1$, an attempt will be made to salvage structures containing unneeded information from the potentially significant extrema lists. If this does not bring the reserve stack up to at least $2 l+1$ structures, an additional $N$ structures are added to the reserve stack using dynamic memory allocation. The parameter $N$ is $2 l$ or larger so that the stack will contain at least $2 l+1$ structures. The memory optimal practice would seem to be to add just enough structures to bring the total to $2 l+1$. It is hoped that using the proposed scheme with a larger value of $N$ will reduce overhead by reducing the number of dynamic storage allocation calls necessary. It is only suboptimal from memory usage by at most $N-1$ structures over the entire run of the jitter calculation. Reducing the number of dynamic storage allocation calls probably has its own storage usage reward as well as reducing execution time. The system must use additional memory to keep track of memory allocated at each call. This additional information would be needed when the system frees up the allocated memory.

This stack management scheme has two important effects: (a) The reserve stack can never become empty. This simplifies the stack maintenance functions. (b) Structures removed from the lists and put back on the reserve stack as a result of Algorithm 1, Step 2, are not reused during the current jitter calculation step update process. This means that structures pointed to by "dominant element" pointers retain their " $\mathrm{y}$ " information and can be queried even if they have been removed from their list and placed on the stack. The program uses this " $y$ " information to determine whether such an structure has, in fact, been removed from the list so that the "dominant element" pointer can be properly updated.

The scheme to salvage unneeded structures from the potentially significant extrema lists is based on the following ideas. If a structure on a potentially significant extrema list contains information which comes from a point in its time series which has been bypassed by the dominant element pointer of the longest window, then it can be returned to the reserve stack. Such a point can be seen at $i=1$ in Fig. 3. As the time series approaches its end, a structure which would need to be kept on a potentially significant extrema list if the time series were to continue indefinitely need not be kept if the information in it is dominated by that in an earlier structure which will never fall off the left end of its window before the end of the time series is reached. Again in Fig. 3, the potentially significant maxima at $i=7$ and 
8 fall into this category. Whenever additional structures are needed on the reserve stack, any of these structures which can be salvaged will be; and the need for allocating additional storage will be reassessed.

This scheme has been realized in a $\mathrm{C}$ code package which integrates jitter analysis with the simulation code which generates the time series and some plot data compression code. Further code provides an interface to MATLAB. All of this code is compiled into a MATLAB MEX-file. In this form, it is part of the PLATSIM, Version 2.0, software package.

\section{Timing results}

Two timing studies are presented. In the first, the time to calculate jitter using the two implementations of Algorithm 1 are compared to each other and to the time to calculate jitter using the easy algorithm. These comparisons are made by calculating jitter in time signals of different lengths. In the second study, experimental verification is sought for the timing formula for Algorithm 1 given in the "Remarks on cost".

\section{Comparison of algorithms and implementations}

The timing studies of the 2 implementations of the jitter calculation algorithm used data from the EOS AM-1 spacecraft [11] as packaged with the PLATSIM software [10]. In this study, there are 7 jitter windows $(m=7)$ of lengths $1,1.8,9,55,420,480$, and 1000 seconds.

Two disturbances were used. The disturbance producing the smaller

jitter analysis problem is called "MODIS scan mirror". The time history of this disturbance extends over 1000 seconds at a sample rate of 200 hertz, so $n=200,001$. The same sample rate is used to produce the 27 outputs to be jitter analyzed. This means that when the 7 jitter windows are in standard position, they cover 201, 361, 1801, 11,001, 84,001, 96,001, and 200,001 points respectively.

The disturbance producing the larger jitter analysis problem is called "MOPITT mirror scan". The time history of this disturbance extends over 1200 seconds at a sample rate of 1000 hertz, so $n=1,200,001$. The same sample rate is used to produce the 27 outputs to be jitter analyzed. This means that when the 7 jitter windows are in standard position, they cover 1001, 1801, 9001, 55,001, 420,001, 480,001, and 1,000,001 points, respectively.

In order to compare execution times of alternative jitter calculations, Algorithm 1 was exercised in both the "Fast Implementation" and the "Memory Conservative Implementation", and jitter was also calculated using the 
"Easy Algorithm". These calculations were made in the context of a PLATSIM time domain analysis. Timing values were obtained using two timing tools. In the case of the "Fast Implementation" and the "Easy Algorithm", the jitter calculation could be isolated from the rest of the code at the MATLAB level, and MATLAB function cputime was used. For all cases, the duration of the total PLATSIM analysis was timed using the UNIX timing function located, in standard implementations of UNIX, in file/usr/bin/time. These total times were compared to times found by running the same data through PLATSIM with the jitter calculation option turned off.

The timing studies were run on a Sun SPARC 10/30 workstation. In an attempt to see how precise the timing numbers are, four of the cases involving the smaller jitter analysis problem were rerun 5 times each on a Sun SPARC 10/512 workstation. It should be noted that since exactly the same problem was run with exactly the same software 5 times, exactly the same amount of calculation was done each time; so the cpu time for the calculation should be the same for each run. However, these were both multi-tasking networked computers. While cputime and /usr/bin/time both purport to measure cpu time for the specific job, they do seem to be influenced by the total load on the computer. Timing runs were made overnights and weekends to try to minimize the effect of other computer workload on the timing measurements, but some multitasking noise, probably from automatically operating system procedures, seems to have affected the timing results.

The cases which were run five times each involved both implementations of Algorithm 1, run both with jitter calculation enabled and with it disabled. Means and standard deviations of total run times for each case were calculated as were the mean and standard deviation of the isolated jitter calculation time in the case of the "Fast Implementation" with jitter enabled. From these calculations, the mean and standard deviation of the time to do jitter related calculations by the two implementations were calculated. The results are given by stating the standard deviation as a per cent of the jitter calculation time. There was an $11 \%$ standard deviation in the time estimate for jitter analysis by the "Fast Implementation" found by taking the difference of the average time for a complete run including jitter calculation and the average time for a complete run excluding jitter calculation. For the "Memory Conservative Implementation", the standard deviation was $41 \%$. Most of this large standard deviation was due to a single data point which was well separated from the cluster formed by the other four points. By removing the obvious outlier from the data set, the standard deviation 
was reduced to $14 \%$. The standard deviation in the directly measured jitter analysis time for the "Fast Implementation" was $7 \%$.

With such a small sample size, these numbers must be considered to be rough approximations. However, it is probably safe to conclude from them that timing numbers presented subsequently are approximately correct in their most significant digit.

Further softening the sharpness of timing data is their dependence on which compiler is used in compiling the executable modules and what optimization levels are called for. The results given in this paper are based on FORTRAN code which was compiled using the Sun "f77" compiler, version V1.4, with optimization flag "-O" and C code which was compiled using the Sun "cc" compiler, version SC1.0, with optimization flag "-O". However, when some of the "Memory Conservative Implementation" tests were rerun using binaries compiled using the GNU "gcc" compiler with optimization flag "-O3", execution times generally improved by 15-20\%.

Table 1 gives the times to calculate jitter using Algorithm 1 in both of its implementations and also doing the calculation using the easy algorithm. The "Easy Algorithm" test was made using FORTRAN 77 subroutines which were compiled using the same optimization flags and linked to MATLAB in the same way as the "Fast Implementation" test.

Table 1: Jitter calculation execution times, seconds

\begin{tabular}{lcc}
\hline \hline Case & $\begin{array}{c}\text { MODIS scan mirror } \\
(n=200,001)\end{array}$ & $\begin{array}{c}\text { MOPITT mirror scan } \\
(n=1,200,001)\end{array}$ \\
\hline $\begin{array}{l}\text { Fast Implementation, } \\
\text { direct measurement }\end{array}$ & $4.8 \times 10^{1}$ & $3.0 \times 10^{2}$ \\
$\begin{array}{l}\text { Fast Implementation, } \\
\text { difference of full runs }\end{array}$ & $1.2 \times 10^{2}$ & $7.5 \times 10^{2}$ \\
$\begin{array}{l}\text { Memory Conservative Implementation, } \\
\text { difference of full runs }\end{array}$ & $2.5 \times 10^{2}$ & $1.9 \times 10^{3}$ \\
$\begin{array}{l}\text { Easy Algorithm, } \\
\text { direct measurement }\end{array}$ & $2.5 \times 10^{5}$ & $1.1 \times 10^{7} \mathrm{a}$ \\
$\begin{array}{l}\text { Easy Algorithm, } \\
\text { difference of full runs }\end{array}$ & $2.5 \times 10^{5}$ & $1.1 \times 10^{7} \mathrm{a}$ \\
\hline \hline
\end{tabular}

$\bar{a}$ estimated

The estimate used for the time to calculate jitter in the big problem using the easy algorithm was arrived at by running this case with only one 
output signal instead of the 27 used in the rest of the cases and multiplying the resulting jitter calculation time by 27 . This estimation was justified experimentally by running the small problem with 1,2 , and 3 outputs. The average time per output varied by about 1 part in 2700 over these three tests. Theoretical considerations support the hypothesis that time to calculate jitter using the easy algorithm is linear in the number of outputs, since the work to calculate jitter using the easy algorithm depends only on the signal length and the window sizes and is independent of the signal shape. Note that the estimated time to do the full problem exceeds 127 days. On the basis of this estimate, Algorithm 1 is claimed to be enabling technology for large jitter problems.

Even considering the imprecision of timing numbers noted previously, some conclusions can be drawn. Note that $n$ for the large case is almost exactly 6 times as large as for the small case. Times for calculating jitter for the large problem using Algorithm 1 are all roughly 6 times the comparable times for the small problem, supporting the claim that, for a given number of windows, the time to calculate jitter using Algorithm 1 is proportional to the number of points in the time series independent of the actual window lengths. The "Memory Conservative Implementation" seems to take about twice as long as the "Fast Implementation". The differences between the "Fast Implementation" times measured directly and as a difference of full runs is at least partly explained by overhead in the PLATSIM program related to outputting the jitter results which was not included in the direct time measurement; but for these two cases, this overhead should have been about the same. Perhaps the discrepancy is due to the imprecision and/or variability of timing measurements.

The improvement over prior technology is clear. For the smaller problem, the speed-up using Algorithm 1 exceeds 3 orders of magnitude, while for the larger problem the improvement in execution time is more like 4 orders of magnitude.

The implications of this on turn-around time for a time domain analysis of the EOS AM-1 spacecraft using PLATSIM are profound. Time domain analysis consisted of simulating the response of the 27 output signals to the disturbance time history, calculating the jitter in these 27 signals, and performing a data compression on these signals so that the PostScript files containing the plots of the output time histories could be made reasonably small. This data compression is done to realize a savings of disk space (from multi-megabytes per file to a few 10's of kilobytes) and time to print the 
hard copies (from about 30 minutes per to more like 2 minutes). The small problem which took almost 3 days to analyze using the old technology was done in less than an hour using the new. The fraction of compute time devoted to jitter analysis dropped from almost $99 \%$ to about $4 \%$ for the fast method and about $7 \%$ for the memory conservative method (and note that the easy algorithm for computing jitter does not have a memory conservative counterpart). For the large problem, the run time decreased from an estimated time of more than 127 days to about 5 hours with jitter analysis time decreasing from over $99.8 \%$ of the total to about $4 \%$ for the fast method and about $9.5 \%$ for the memory conservative method.

\section{Parameter Dependency}

Theoretical analysis suggests that the time to compute jitter using Algorithm 1 should be of the form $(a m+b) n$ where $n$ is the number of points in the time series, $m$ is the number of windows, and $a$ and $b$ are constants independent of all the problem parameters, specifically including the actual window lengths. (The " $\mathrm{cm}+d$ " part of the timing formula given in the earlier "Remarks on cost" is in the noise level of the computer execution timing instrument, and will be ignored in this study.)

A timing study was run to measure how the jitter calculation time using Algorithm 1 depends on $n$ and $m$, and to see if it is independent of $w_{1}, \ldots, w_{m}$ (the individual window lengths). The study involved 3808 individual cases. The time series length $n$ ranged from 100,001 to 1,200,001 in increments of 100,000 . The time history which provided the input data was the MODIS roll output from the EOS AM-1 simulation using the same MOPITT mirror scan disturbance used for the larger jitter analysis problem in the previous subsection. For $n<1200001$, the first $n$ points were used. The basic window set was scaled by each of the factors $0.2,0.6,1.0$, and 1.3; any windows longer than the truncated time series were discarded; and the jitter calculation was done with each subset of the resulting window set. This gave values of $m$ ranging from 1 to 7 and 26 usable window lengths distributed in a fairly uniform exponential manner from 0.2 seconds to 1000 seconds.

These parameter dependency timing tests were made using the "Fast Implementation" of Algorithm 1. The test data had been previously calculated and saved for use in these tests. Timing studies were run directly from MATLAB and not in the context of a PLATSIM time domain analysis. Times were measured using MATLAB's cputime function. Three timing 
runs were made on each of the 3808 test cases.

The relevant data from the $i$-th case are $n_{i}$, the number of points in the time series, $m_{i}$, the number of windows, and $\tau_{i}$, the time to calculate jitter. The dependent variable was taken to be $z_{i}$, defined to be $\tau_{i} / n_{i}$, and the dependence of $z_{i}$ on $m_{i}$ was investigated. A scatter plot of the points $\left(m_{i}, z_{i}\right)$ showed that the data tended to concentrate along a straight line, but a few outliers were observed. Further analysis showed that any case which gave an outlier in one of the three timing runs did not produce an outlier in either of the other two runs. It was concluded that the outliers resulted from external influences on the timing instrument. This was taken as justification that the outliers could be eliminated.

Outliers were eliminated by taking $z_{i}$ to be the median value of the ratios $\tau_{i} / n_{i}$ formed with data from each of the 3 runs. A scatter plot of $\left(m_{i}, z_{i}\right)$ now concentrated along a straight line with no wild points.

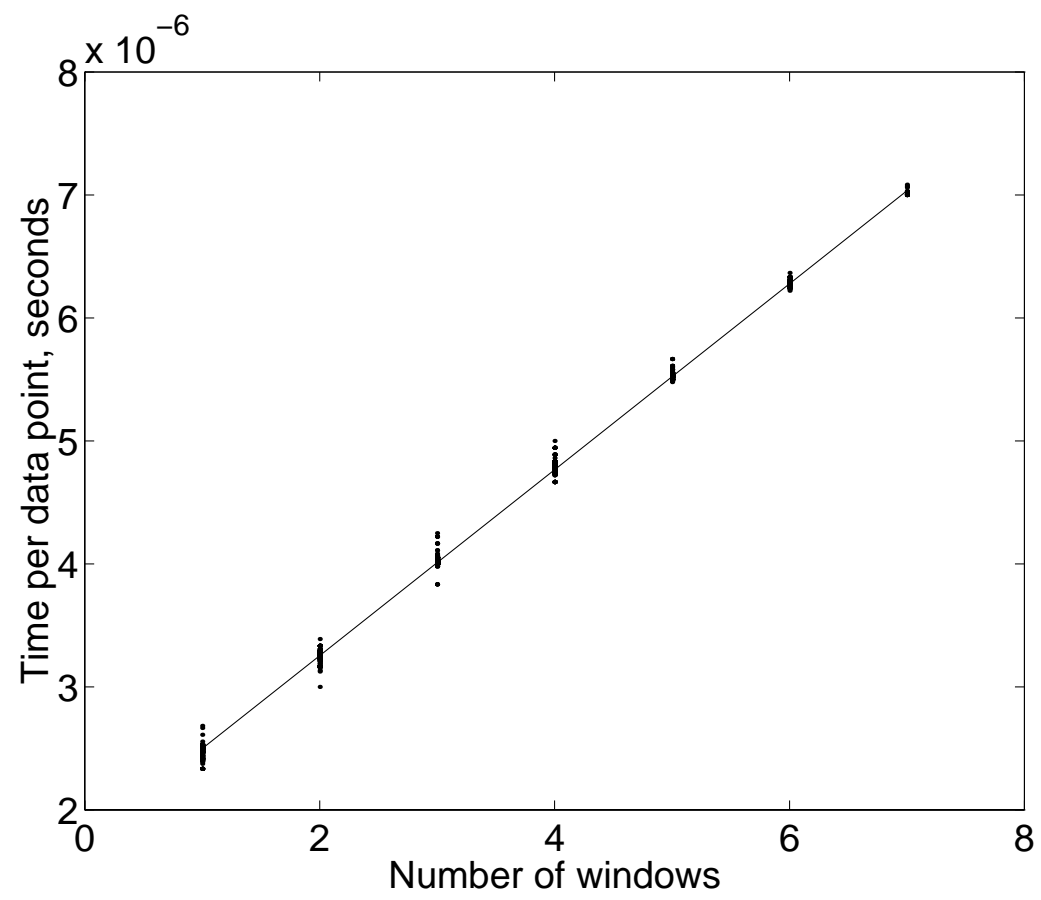

Figure 4: Linear regression fit to window size timing data

Using linear least squares regression on this data, it was found that $z_{i} \approx a m_{i}+b$ with $a=7.56 \times 10^{-7} \mathrm{sec}$ and $b=1.74 \times 10^{-6} \mathrm{sec}$. The 
root mean square error in this approximation was $2.73 \times 10^{-8}$ sec which was about $0.6 \%$ of the mean $z_{i}$ value of $4.27 \times 10^{-6}$ sec. This is a tight fit which experimentally validates the theoretical timing formula. A scatter plot of the median computational time per time series point as a function of the number of windows is shown in Fig. 3 together with the least squares linear regression fit line.

Estimating jitter computation time as $(a m+b) n$, it is seen that the time to calculate jitter with respect to one window is about $(a+b) n$ while each additional window adds about an to the time. This means that the time to calculate jitter with respect to each window after the first is about $30 \%$ of the time to calculate jitter with respect to the first window. The conclusion to be drawn from this is that it is substantially more efficient to calculate jitter over several windows simultaneously than to do the calculations for each window individually.

\section{Memory Conservation Results}

In the small problem, $n=200,001$ and $l=27$. Thus, it takes $200001 \times$ $27 \times 8$ bytes of memory to store the time histories of the outputs in 8 byte double precision words. The jitter computation also requires 2 integer arrays of length $n$ to hold the information for the lists of potentially significant local extrema. Assuming that an integer occupies 4 bytes of storage, and recalling that a megabyte of memory refers to $2^{20}$ bytes, it is seen that the major data storage part of the memory requirement for the "Fast Implementation" of doing the jitter calculation is almost 43 megabytes.

The storage structure used in the "Memory Conservative Implementation" must hold one double precision number $\left(y\left(t_{i}\right)\right)$, one integer $(i)$, and two pointers. In the implementation of the $\mathrm{C}$ programming language used in this study (the standard C compiler cc furnished with the Sun OS 4.x), these individual fields occupy 8, 4, 4, and 4 bytes respectively. However, the system uses 24 bytes for the complete structure. One can only speculate about those 4 extra bytes. Perhaps it has something to do with aligning structures on word boundaries.

The choice was made to allocate these structures in blocks of 10,000. The small problem required 4 of these blocks. Thus, the major data storage part of the memory requirement for the "Memory Conservative Implementation" of doing the jitter calculation was $1000 \times 24 \times 4$ bytes which is less than 1 megabyte. The memory savings here were almost $97.9 \%$. 
The large problem has $n=1,200,001$ and $l=27$. Repeating the previous calculation with these numbers, it is seen that the major data storage part of the memory requirement for the "Fast Implementation" of doing the jitter calculation is over 256 megabytes. When it was run with the "Memory Conservative Implementation", 9 blocks of structures were allocated, so the major data storage part of the memory requirement for jitter analysis in this run was barely over 2 megabytes. In this case, the memory savings were over $99.2 \%$.

It should be noted that the memory usage for the "Fast Implementation" is completely determined by the length and number of time signals being analyzed, but that the memory usage for the "Memory Conservative Implementation" also depends on the signal shape and the lengths of the jitter windows. An extremely noisy signal would tend to require less memory while a signal with long monotone stretchs would require more. So, while the numbers given here for the "Memory Conservative Implementation" are indicative of the savings, results will vary somewhat from case to case.

\section{Conclusions}

A fast algorithm for calculating jitter in a time signal has been presented. When applied to large problems typical of those found in spacecraft design analysis, it computes the NASA metric of jitter faster than prior technology by 3 to 4 orders of magnitude. In the case of very large problems, this is enabling technology. In any case, it tames the jitter analysis calculation so that instead of being the overwhelmingly dominant element in the total calculation time of a typical analysis, it is a relatively small part.

Two implementations of the algorithm have been described. The faster one takes advantage of knowledge of the entire time series when this information is available. The memory conservative version allows the calculation to proceed with much less memory usage, preventing excessive page swapping and even disk swap space overflow for particularly large problems. If jitter must be calculated with respect to several windows, it is much more efficient to calculate the jitter for all the windows simultaneously than it is to do the calculation for each window independently of the others. 


\section{References}

[1] Daniel P. Giesy. Efficient calculation of a jitter/stability metric. AIAA Journal of Spacecraft and Rockets, 34(4):549-557, July-August 1997.

[2] Bolek, J. T. private communication, Feb. 1997.

[3] Maghami, P. G. private communication, Mar. 1997.

[4] Neste, S. L. UARS pointing error budget, (CDRL 220.04). Program Information Release U_1K21_UARS_517, GE Space Division, July 1986.

[5] Ford, T. EOS pointing error budget, prediction and verification concept. Technical Report EOS-DN-SE\&I-043 Rev. A, Martin Marietta Astro Space, August 271993.

[6] Ram, M. and Throckmorton, A. EOS instrument jitter assessment and mitigation AIAA 93-1004. In Aerospace Design Conference, Irvine, CA. AIAA/AHS/ASEE, Feb 1993.

[7] Belvin, W. K., Maghami, P. G., Tanner, C., Kenny, S. P., and Cooley, V. Evaluation of CSI enhancements for jitter reduction on the EOS AM-1 observatory. In L. Meirovitch, editor, Dynamics and Control of Large Structures, Proceedings of the Ninth VPI\&SU Symposium, pages 255-266, Blacksburg, VA, May 1993. Virginia Polytechnic Institute and State University.

[8] Ropbeck, L. S., Rathbun, D. B., and Lehman, D. H. Precision pointing copntrol for an orbinal earth observing system. IEEE Control Systems Magazine, 11(3):46-52, 1991.

[9] Maghami, P. G., Kenny, S. P., and Giesy, D. P. PLATSIM: A simulation and analysis package for large-order flexible systems. TM, NASA Langley Research Center, Hampton, VA 23681, 1996. To appear as a NASA Langely Research Center Technical Memorandum.

[10] Maghami, P. G., Kenny, S. P., and Giesy, D. P. PLATSIM: An efficient linear simulation and analysis package for large-order flexible systems. TP 3519, NASA Langley Research Center, Hampton, VA 23681, August 1995. 
[11] Maghami, P. G., Kenny, S. P., and Giesy, D. P. The PLATSIM software package: A simulation and analysis tool for large-order flexible systems with applications to EOS AM-1. In Robert D. Culp and James D. Medbery, editors, Guidance and Control 1995, volume 88 of Advances in the Astronautical Sciences, pages 39-57, San Diego, CA, 1995. American Astonautical Society, Univelt, Inc. Proceedings of the annual AAS Guidance and Control Conference held February 1-5, 1995, Keystone, CO. 